DIIS - I3A

C/ María de Luna num. 1

E-50018 Zaragoza

Spain

Internal Report: 2007-V05

\title{
Homography-Based Visual Control of Nonholonomic Vehicles
}

G. López-Nicolás, C. Sagüés and J.J. Guerrero

If you want to cite this report, please use the following reference instead:

Homography-Based Visual Control of Nonholonomic Vehicles, G. López-Nicolás, C. Sagüés and J.J. Guerrero, IEEE Int. Conference on Robotics and Automation, pages 1703-1708, Rome - Italy, April 2007. 


\title{
Homography-Based Visual Control of Nonholonomic Vehicles
}

\author{
G. López-Nicolás, C. Sagüés and J.J. Guerrero \\ DIIS - I3A, Universidad de Zaragoza, Spain \\ \{gonlopez, csagues, jguerrer\}@unizar.es
}

\begin{abstract}
This paper presents a new visual control approach based on homography. The method is intended for nonholonomic vehicles with a fixed monocular system on board. The idea of visual control used here is the usual approach where the desired position of the robot is given by a target image taken at that position. This target image is the only previous information needed by the control law to perform the navigation from the initial position to the target. The control law is designed by the input-output linearization of the system using elements of the homography as output. The contribution is a controller that deals with the nonholonomic constraints of the mobile platform needing neither decomposition of the homography nor depth estimation to the target.
\end{abstract}

\section{INTRODUCTION}

Visual control, also called visual servoing, is a very extensive and mature field of research where many important contributions have been presented in the last decade [4], [5], [12], [14], [18]. Two interesting surveys on this topic are [6] and [9].

The framework of the method presented here is a mobile robot with nonholonomic constraints with a fixed camera on board. The idea of the visual control method is to drive the robot from an initial position to the target. The desired position is given by an image previously taken at the target position, and using the images taken during the navigation the robot is led to the target.

A traditional approach is to perform the motion by computing the epipolar geometry between the current image and the target one [1], [11], [15]. Nevertheless, the estimation of the epipolar geometry becomes ill conditioned for planar scenes, this is a drawback of these approaches because planar scenes are usual in human environments. A natural way to overcome this problem is by using the homography model. In [13] it is proposed a method based on the estimation of the homography matrix related to a virtual plane attached to an object. This method provides a more stable estimation when the epipolar geometry degenerates. In [3] a system for car platooning using visual tracking is presented by estimating the homography between a selected reference template attached to ta leading vehicle. A significant issue with monocular camera-based vision systems is the lack of depth information. Fang et al. [7] proposed the asymptotic regulation of the position and orientation of a mobile robot by exploiting homography-based visual servo control strategies. Thus, the homography is decomposed and orientation and scaled Euclidean position can be obtained; the unknown

This work was supported by projects DPI2006-07928, IST-1-045062URUS-STP. time-varying depth information is related to a constant depthrelated parameter.

These homography-based methods usually require the decomposition of the homography, which is not a trivial issue. Two examples of approaches which do not use the homography decomposition are [16] which is based on a 2D homography and [2] which presents an uncalibrated approach for manipulators. Nevertheless, methods like these are usually not intended for nonholonomic robots.

The method presented in this paper is based on the input-output linearization of the system considering the nonholonomic constraints of the platform. A main feature of this approach is the design of the input control based on the homography elements directly. Then, the visual control problem is transformed in a tracking problem where the desired values of these homography elements during the motion are defined. This approach needs neither homography decomposition nor depth estimation.

The paper is divided as follows: Section II presents the homography, developing its elements as a function of the system parameters to be used in the control law design. Section III describes the motion model, followed by the design of the control law in Section IV. Experimental evaluation is given in Section V showing the method performance with noise and with calibration errors. Section VI gives the conclusions.

\section{Perceptual Model}

Two perspective images can be geometrically linked by a homography. This homography relates points in one image belonging to a plane of the scene to the corresponding points in the other image (Fig. 1). The homography between two images can be computed using image point matches [8].

Let us suppose the two images are obtained with the same camera, whose projection matrixes in a common reference system are $\mathbf{P}_{1}=\mathbf{K}[\mathbf{I} \mid \mathbf{0}]$ and $\mathbf{P}_{2}=\mathbf{K}[\mathbf{R} \mid \mathbf{t}]$, with $\mathbf{t}=-\mathbf{R c}$, being $\mathbf{R}$ the camera rotation, $\mathbf{c}$ the distance between the cameras, and $\mathbf{K}$ the internal calibration matrix, defined as

$$
\mathbf{K}=\left[\begin{array}{ccc}
\alpha_{x} & s & x_{0} \\
0 & \alpha_{y} & y_{0} \\
0 & 0 & 1
\end{array}\right]
$$

where $\alpha_{x}$ and $\alpha_{y}$ are the focal length of the camera in pixel dimensions; $s$ is the skew parameter and $\left(x_{0}, y_{0}\right)$ are the coordinates of the principal point. We have that $\alpha_{x}=f m_{x}$ and $\alpha_{y}=f m_{y}$, where $f$ is the focal length and $m_{x}, m_{y}$ are the pixels per distance unit. In practice, we assume that 


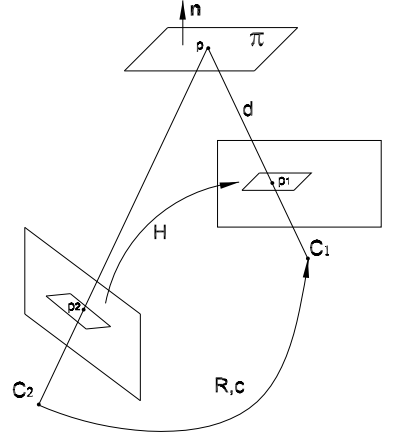

(a)

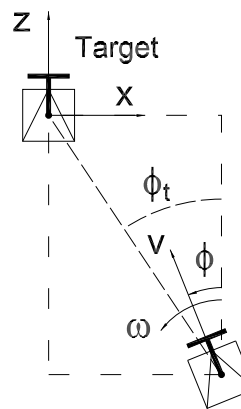

(b)
Fig. 1. (a) Homography from a plane between two views, where $C_{1}$ and $\mathrm{C}_{2}$ are the optical centres of the cameras. (b) Coordinate system.

the principal point is in the center of the image $\left(x_{0}=0\right.$, $\left.y_{0}=0\right)$ and that there is no skew $(s=0)$.

A point can be represented in the image with homogeneous coordinates as $\mathbf{p}=(x, y, 1)^{T}$. A projective transformation $\mathbf{H}$ relates pairs of matched points belonging to a plane of the scene $(\pi)$ in such a way that $\mathbf{p}_{2}=\mathbf{H} \mathbf{p}_{1}$.

A homography $\mathbf{H}$ can be related to camera motion in such a way that

$$
\mathbf{H}=\mathbf{K}\left(\mathbf{R}-\mathbf{t} \frac{\mathbf{n}^{T}}{d}\right) \mathbf{K}^{-1}=\mathbf{K R}\left(\mathbf{I}+\mathbf{c} \frac{\mathbf{n}^{T}}{d}\right) \mathbf{K}^{-1},
$$

where $\mathbf{n}=\left(n_{x}, n_{y}, n_{z}\right)^{T}$ is the normal to the scene plane that generates the homography and $d$ is the distance to the plane from the origin of the global reference (Fig. 1).

We consider a mobile robot moving in a planar surface (Fig. 1); in this case the location of the robot is defined by the vector $(x, z, \phi)$. Therefore the planar motion constraint yields:

$$
\mathbf{R}=\left[\begin{array}{ccc}
\cos \phi & 0 & \sin \phi \\
0 & 1 & 0 \\
-\sin \phi & 0 & \cos \phi
\end{array}\right] \text { and } \mathbf{c}=(x, 0, z)^{T}
$$

Therefore, the homography corresponding to a planar motion scheme is:

$$
\mathbf{H}=\left[\begin{array}{ccc}
h_{11} & h_{12} & h_{13} \\
0 & 1 & 0 \\
h_{31} & h_{32} & h_{33}
\end{array}\right]
$$

The homography is normalized by dividing $\mathbf{H} / h_{22}$, given that $h_{22}$ is never zero due to the constraint of planar motion. Developing expression (1) we obtain the homography elements as a function of the camera and motion parameters:

$$
\left\{\begin{array}{l}
h_{11}=\cos \phi+(x \cos \phi+z \sin \phi) \frac{n_{x}}{d} \\
h_{12}=\frac{\alpha_{x}}{\alpha_{y}}(x \cos \phi+z \sin \phi) \frac{n_{y}}{d} \\
h_{13}=\alpha_{x}\left(\sin \phi+(x \cos \phi+z \sin \phi) \frac{n_{z}}{d}\right) \\
h_{31}=\frac{1}{\alpha_{x}}\left(-\sin \phi+(-x \sin \phi+z \cos \phi) \frac{n_{x}}{d}\right) \\
h_{32}=\frac{1}{\alpha_{y}}(-x \sin \phi+z \cos \phi) \frac{n_{y}}{d} \\
h_{33}=\cos \phi+(-x \sin \phi+z \cos \phi) \frac{n_{z}}{d}
\end{array}\right.
$$

The analysis of these elements of the homography will lead to the design of the visual servoing controller.

\section{Robot Motion Model}

Let us suppose a nonholonomic differential kinematics expressed in a general way as

$$
\begin{aligned}
\dot{\mathbf{x}} & =\mathbf{f}(\mathbf{x}, \mathbf{u}) \\
\mathbf{y} & =\mathbf{h}(\mathbf{x})
\end{aligned}
$$

where $\mathbf{x}=(x, z, \phi)^{T}$ denotes the state vector, $\mathbf{u}=(v, \omega)^{T}$ the input vector, and $\mathbf{y}$ the output vector. The particular nonholonomic differential kinematics of the robot expressed in state space as a function of the translation and rotation velocities of the robot $(v, \omega)$ is as follows

$$
\begin{gathered}
\left(\begin{array}{c}
\dot{x} \\
\dot{z} \\
\dot{\phi}
\end{array}\right)=\left(\begin{array}{c}
\sin \phi \\
\cos \phi \\
0
\end{array}\right) v+\left(\begin{array}{l}
0 \\
0 \\
1
\end{array}\right) \omega \\
\mathbf{y}=\left(h_{11}, h_{12}, \ldots h_{33}\right)^{T} .
\end{gathered}
$$

\section{INPUT-OUTPUT LINEARIZATION SCHEME}

The approach presented performs the robot navigation by controlling the elements of the homography. Then, the problem of visual servoing is transformed into a tracking problem where the desired values of the homography elements along the motion are defined.

\section{A. Input-Output Linearization}

We have a nonlinear system relating inputs and outputs. A linearization is developed by differentiating the homography elements until we can solve for the control inputs. Linearization by differentiating is a common way to face the design of nonlinear control systems [10], [17].

As the system has two variables to be controlled $(v, \omega)$, at least two elements of the homography are needed to guarantee the controllability. Elements $h_{21}, h_{22}$ and $h_{23}$ do not give information because of the planar motion constraint. Elements $h_{31}$ and $h_{32}$ are discarded because they are small and sensitive to noise compared with the rest of the elements, this is because of the factors $1 / \alpha_{x}$ and $1 / \alpha_{y}$ appearing in $h_{31}$ and $h_{32}$ respectively. In our monocular system, planes in front of the robot with dominant $n_{z}$ will be detected more easily. Thus, from the elements left, we have selected those depending on $n_{z}$ (i.e. $h_{13}$ and $h_{33}$ ).

Note that the normal vector of the plane $(\mathbf{n})$ and the distance between the plane and the origin $(d)$ are referred to the global reference attached to the target position. Given that the target is fixed, these parameters $(\mathbf{n}, d)$ are constant, and its derivative is zero. The derivatives of $h_{13}$ and $h_{33}$ with respect to the time gives

$$
\begin{aligned}
& \dot{h}_{13}=2 \alpha_{x} \sin \phi \cos \phi \frac{n_{z}}{d} v+\alpha_{x} h_{33} \omega \\
& \dot{h}_{33}=\left(\cos ^{2} \phi-\sin ^{2} \phi\right) \frac{n_{z}}{d} v-\frac{h_{13}}{\alpha_{x}} \omega
\end{aligned}
$$

These derivatives have been simplified by using the next relations, which come from different combinations between the elements of the homography (3),

$$
\begin{aligned}
& (x \cos \phi+z \sin \phi)=\left(\frac{h_{13}}{\alpha_{x}}-\sin \phi\right) \frac{d}{n_{z}} \\
& (-x \sin \phi+z \cos \phi)=\left(h_{33}-\cos \phi\right) \frac{d}{n_{z}}
\end{aligned}
$$




\section{B. Control Law}

After the first derivative we have already got a linear relation between the system input and output. From (6) we have the necessary equations from the elements $h_{13}$ and $h_{33}$ of the homography to be used in the control law. Thus, $\mathbf{y}=\left(h_{13}, h_{33}\right)^{T}$, and the control law is:

$$
\left(\begin{array}{c}
\nu_{13} \\
\nu_{33}
\end{array}\right)=\mathbf{L}\left(\begin{array}{c}
v \\
\omega
\end{array}\right),
$$

where the decoupling matrix is

$$
\mathbf{L}=\left[\begin{array}{cc}
\alpha_{x} \sin (2 \phi) \frac{n_{z}}{d} & \alpha_{x} h_{33} \\
\cos (2 \phi) \frac{n_{z}}{d} & -\frac{h_{13}}{\alpha_{x}}
\end{array}\right] .
$$

Solving for the control outputs we have

$$
\left(\begin{array}{c}
v \\
\omega
\end{array}\right)=\mathbf{L}^{-1}\left(\begin{array}{c}
\nu_{13} \\
\nu_{33}
\end{array}\right),
$$

where the control matrix is

$$
\mathbf{L}^{-1}=\frac{\left[\begin{array}{cc}
\frac{h_{13}}{\alpha_{x}} \frac{d}{n_{z}} & \alpha_{x} h_{33} \frac{d}{n_{z}} \\
\cos (2 \phi) & -\alpha_{x} \sin (2 \phi)
\end{array}\right]}{\sin (2 \phi) h_{13}+\alpha_{x} h_{33} \cos (2 \phi)} .
$$

The new inputs of the control $\left(\nu_{13}, \nu_{33}\right)^{T}$ are given as a function of the current values of the homography elements $\left(h_{13}, h_{33}\right)^{T}$ and their desired values $\left(h_{13}^{d}, h_{33}^{d}\right)^{T}$ which are the trajectories to be followed [17],

$$
\left(\begin{array}{c}
\nu_{13} \\
\nu_{33}
\end{array}\right)=\left(\begin{array}{c}
\dot{h}_{13}^{d}-k_{13}\left(h_{13}-h_{13}^{d}\right) \\
\dot{h}_{33}^{d}-k_{33}\left(h_{33}-h_{33}^{d}\right)
\end{array}\right) .
$$

where $k_{13}$ and $k_{33}$ are the gains of the control. Note that the parameter $\frac{d}{n_{z}}$, which is constant, appears in the first row of $\mathbf{L}^{-1}$ and then it can be considered as a gain of the corresponding output. The decoupling matrix of the control needs the values of $\alpha_{x}, h_{13}, h_{33}$ and $\phi$. As the homography is computed directly from the images, the elements $h_{13}$ and $h_{33}$ are known. The focal length $\alpha_{x}$ is given by the camera calibration and $\phi$ can be computed as it follows. From the parallax relative to the plane that induces the homography, with at least two points not belonging to the plane (Appendix A), the epipole in the target $\left(e_{t x}\right)$ and current $\left(e_{c x}\right)$ images give $\phi=\arctan \left(\frac{e_{c x}}{\alpha_{x}}\right)-\arctan \left(\frac{e_{t x}}{\alpha_{x}}\right)$.

When the current and target positions are the same up to a rotation, the parallax is bad conditioned. However, in this case the value of $\phi$ can be computed directly from the eigenvalues of the homography, which are $\left(1, e^{i \phi}, e^{-i \phi}\right)$. Alternatively $\phi$ can be computed from trace $(H)=1+2 \cos \phi$. We can detect easily when the robot is close to the target by measuring the parallax of the points not belonging to the plane of the homography.

We need $\mathbf{L}$ to be invertible. Thus, it must be

$$
\operatorname{det}(\mathbf{L})=-\sin (2 \phi) h_{13} \frac{n_{z}}{d}-\alpha_{x} \cos (2 \phi) h_{33} \frac{n_{z}}{d} \neq 0 .
$$

We can use the expressions (3) in order to have the determinant as a function of $x, z$ and $\phi$ :

$$
\cos \phi+(x \sin \phi+z \cos \phi) \frac{n_{z}}{d} \neq 0,
$$

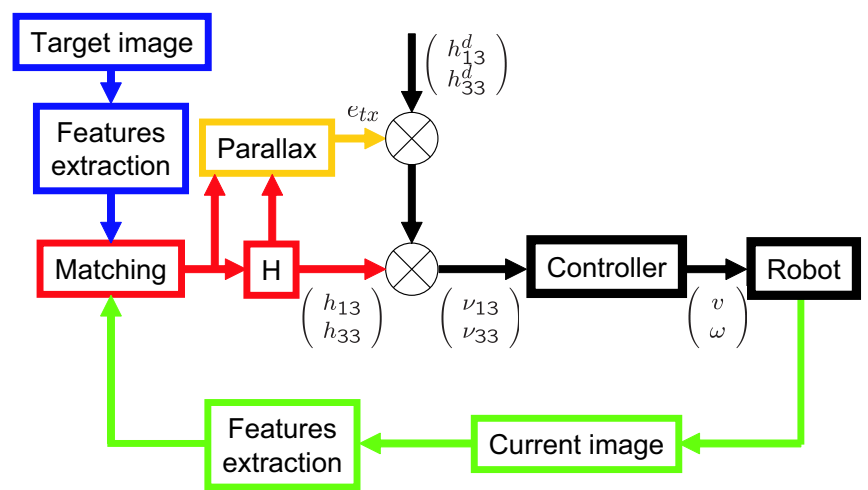

Fig. 2. Diagram of the control loop.

and using $x=-z \tan \phi_{t}$, where $\phi_{t}$ is the angle that relates the current position with the target one, $\mathbf{L}$ is invertible if:

$$
\cos \phi \cos \phi_{t}+\cos \left(\phi+\phi_{t}\right) z \frac{n_{z}}{d} \neq 0 .
$$

Supposing the target is in the field of view of the current camera, for example between $\pm 40^{\circ}$, we have that $\cos \phi>0$ and $\cos \phi_{t}>0$. If the current position of the robot is behind the target, then $z<0$ (Similarly, if the robot starts with the target behind, it will move backwards, having $z>0$ and $\cos \phi_{t}<0$ ). The plane that produces the homography is visible for the cameras, so $n_{z}<0$ in our reference. Therefore the previous determinant is always positive if $\left|\phi+\phi_{t}\right|<\pi / 2$. Because of the camera field-of-view constraint we need to consider that the lateral distance to compensate is smaller than the depth distance, otherwise the scene shared by the cameras could leave the field of view. Then, we conclude that the determinant of the control matrix is never equal to zero in the field of work and therefore it is not singular.

The control loop of the approach presented is shown in the diagram of Fig. 2. An image in the current position is taken at each loop of the control. The homography that links it with the target image can be computed from the feature matching. Having the homography, the control performs a tracking of the desired trajectories of the homography elements, giving the velocities of the robot. When the loop finishes, the robot is in the target position, current and target images are the same, and the homography is the identity matrix.

\section{Desired Trajectories of the Input Control}

The motion performed by the robot depends on how we select the desired trajectories of the homography elements used in the control $\left(h_{13}^{d}, h_{33}^{d}\right)$. Basically, we can relate $h_{33}$ with the motion along $z$-axis and $h_{13}$ with the robot orientation. Then, if we had considered a holonomic platform, position and orientation error could be corrected independently. The problem that arises with nonholonomic constraints is to define the trajectories $h_{13}^{d}$ and $h_{33}^{d}$ required to correct properly the lateral distance to the target. The selected trajectory for $h_{33}$ is a smooth function that converges to 1 guaranteeing that the depth distance is corrected. The desired trajectory of $h_{13}$ can be divided in two phases by time $T_{1}$. If $t \leq T_{1}$ the robot is driven to get a proper orientation in order to allow 


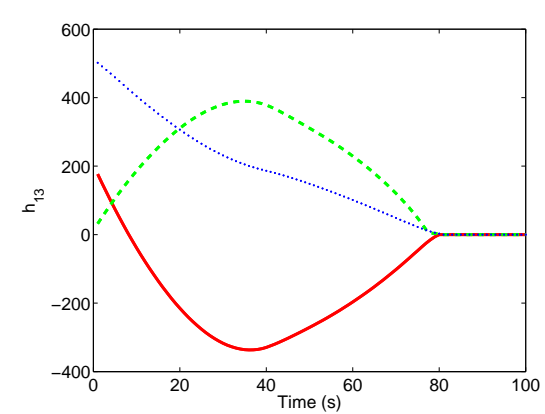

(a) $h_{13}$

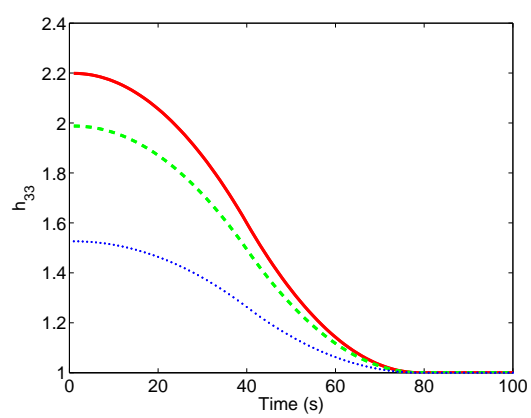

(b) $h_{33}$

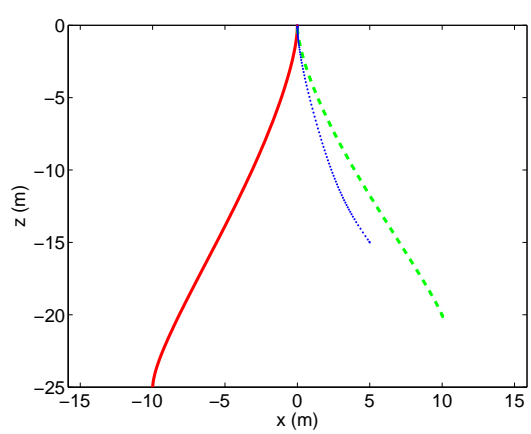

(c) Paths

Fig. 3. (a-b) Examples of the evolution of $h_{13}^{d}$ and $h_{33}^{d}$ obtained for different initial positions and its correspondent paths (c).

a parabolic motion to the target. With $t>T_{1}$ we need to ensure that $h_{13}^{d}$ evolve accordingly to $\phi_{t}(t)$ in such a way that lateral and orientation error are corrected simultaneously. The functions proposed are continuous and time differentiable. The set of desired trajectories to be tracked are defined as:

If $0 \leq t \leq T_{1}$,

$\left\{\begin{array}{l}h_{13}^{d}(t)=\left(h_{13}(0)-g_{t}\right)\left(\frac{t^{2}}{T_{1}^{2}}-2 \frac{t}{T_{1}}+1\right)+g_{t} \\ h_{33}^{d}(t)=\left(\frac{1-h_{33}(0)}{2}\right)\left(\frac{t^{2}}{T_{1}^{2}}+1\right)+\left(3 h_{33}(0)-1\right) / 2\end{array}\right.$

If $T_{1}<t \leq T_{2}$,

$\left\{\begin{array}{l}h_{13}^{d}(t)=h_{13}\left(T_{1}\right) \frac{\phi_{t}(t)}{\phi_{t}\left(T_{1}\right)} \\ h_{33}^{d}(t)=\left(\frac{h_{33}(0)-1}{2}\right)\left(\frac{\left(t-T_{1}\right)^{2}}{\left(T_{2}-T_{1}\right)^{2}}-2 \frac{t-T_{1}}{T_{2}-T_{1}}+1\right)+1\end{array}\right.$

If $t>T_{2}$,

$$
\left\{\begin{array}{l}
h_{13}^{d}(t)=0 \\
h_{33}^{d}(t)=1
\end{array}\right.
$$

where $g_{t}=\alpha_{x} \sin \left(k_{t} \phi_{t}\right)$. The constant parameter $k_{t}$ represents the curvature of the path followed. If $k_{t}=1$ the robot will follow a straight line path to the target, a higher value will produce more rotation at the beginning of the motion (i.e. $k_{t}>3$ ), then the lateral error will be corrected faster but the scene could get out of the field of view of the camera. In our simulations we have selected $k_{t}=1.5$. The variable $\phi_{t}$, which is the angle of the straight line joining the current position of the robot with the target position, can be computed using the plane parallax (Appendix A) as $\phi_{t}=-\arctan \left(e_{t x} / \alpha_{x}\right)$.

The desired functions depend on the homography and then they depend on the initial position. As the robot moves, the previous equations adapt the evolution of the homography desired values guaranteeing the convergence to the target. Several examples for different initial positions are shown in Fig. 3. The starting positions are $\left(-10,-25,-5^{\circ}\right)$, $\left(10,-20,15^{\circ}\right)$ and $\left(5,-15,35^{\circ}\right)$ while the target is in all the cases $\left(0,0,0^{\circ}\right)$. The desired trajectories $h_{13}^{d}$ and $h_{33}^{d}$ are shown in Fig. 3 (a) and (b) respectively. The trajectories of $h_{13}$ and $h_{33}$ obtained are the same as the desired ones. The resulting path is shown in Fig. 3(c), each example is represented with a different line style. In these simulations the values of $T_{1}$ and $T_{2}$ have been selected as $40 \mathrm{~s}$ and $80 \mathrm{~s}$ respectively.

\section{Stability Analysis}

In order to analyze the stability of the proposed control, we define the next Lyapunov function:

$$
V=V_{z}+V_{\phi}=\left(z(t)-z^{d}\right)^{2} / 2+\left(\phi(t)-\phi^{d}\right)^{2} / 2 .
$$

This function is related with the control parameters (3) and is positive definite taking into account the desired trajectories to be tracked. Now we analyze this function in each interval of time and in each quadrant of our reference, having the target in the origin. In $1^{\text {st }}$ and $2^{\text {nd }}$ quadrant the target is behind the initial position and the robot moves backwards, and in $3^{r d}$ and $4^{\text {th }}$ quadrant the target is in front of the initial position. After differentiating the Lyapunov candidate function we have

$$
\dot{V}=\dot{V}_{z}+\dot{V}_{\phi}=\left(z-z^{d}\right) v \cos \phi+\left(\phi-\phi^{d}\right) \omega .
$$

The analysis of each term of $\dot{V}$ to show it is negative is summarized in Table I. For this analysis we need to study the sign of the velocities given by the control law (9),

$$
\left\{\begin{array}{l}
v=\frac{h_{13} d \nu_{13} /\left(\alpha_{x} n_{z}\right)+\alpha_{x} h_{33} d \nu_{33} / n_{z}}{\sin (2 \phi) h_{13}+\alpha_{x} h_{33} \cos (2 \phi)} \\
\omega=\frac{\cos (2 \phi) \nu_{13}-\alpha_{x} \sin (2 \phi) \nu_{33}}{\sin (2 \phi) h_{13}+\alpha_{x} h_{33} \cos (2 \phi)}
\end{array}\right.
$$

The analysis on the signs of the previous expressions lead to the confirmation of $\dot{V}<0$. For this task we also have to study the sign of the homography elements used in the control (3), knowing that $x=-z \tan \phi_{t}$,

$$
\left\{\begin{array}{l}
h_{13}=\frac{\alpha_{x}}{\cos \phi_{t}}\left(\sin \phi \cos \phi_{t}+z \sin \left(\phi-\phi_{t}\right) \frac{n_{z}}{d}\right) \\
h_{33}=\frac{1}{\cos \phi_{t}}\left(\cos \phi \cos \phi_{t}+z \cos \left(\phi+\phi_{t}\right) \frac{n_{z}}{d}\right)
\end{array}\right.
$$

The procedure followed is similar to the presented in Table I, and it is not shown here. Taking this into account, the control of the system is stable in the Lyapunov sense. The desired functions to be tracked are bounded by definition, and the inputs designed for the tracking controller (10) are known to represent an exponentially stable error dynamics [17], so the tracking error converges to zero exponentially.

It is known that $(x, z, \theta)=(0,0,0) \Leftrightarrow \mathbf{H}=\mathbf{I}$. Using our control, we have at the end of motion $h_{13}=0$ and $h_{33}=1$. Given that $h_{13}^{d}=h_{13}=0$, from the tracked trajectories, we have $h_{13}^{d} \sim \phi_{t}$, and therefore $x=0$ (see Fig. 1). Taking this into account, we need to prove that the final pose is 
TABLE I

ANALYSIS OF EACH TERM OF THE DERIVATIVE LYAPUNOV CANDIDATE FUNCTION (13) TO SHOW IT IS STRICTLY NEGATIVE.

\begin{tabular}{|l|c|c|}
\hline & Quadrant 1,3 & Quadrant 2,4 \\
\hline$\dot{V}_{\phi}\left(t \leq T_{1}\right)=\left(\phi-\phi^{d}\right) \omega$ & $\phi-\phi^{d}>0$ & $\phi-\phi^{d}<0$ \\
$\left|\phi^{d}\right|>|\phi|$ & $w<0$ & $w>0$ \\
\hline$\dot{V}_{\phi}\left(t>T_{1}\right)=\phi \omega$ & $\phi<0, w>0$ & $\phi>0, w<0$ \\
\hline \hline & Quadrant 1,2 & Quadrant 3,4 \\
\hline$\dot{V}_{z}\left(t \leq T_{1}\right)=\left(z-z^{d}\right) v \cos \phi$ & $z-z^{d}>0$ & $z-z^{d}<0$ \\
$\left|z^{d}\right|<|z|$ & $v<0$ & $v>0$ \\
\hline$\dot{V}_{z}\left(t>T_{1}\right)=z v \cos \phi$ & $z>0, v<0$ & $z<0, v>0$ \\
\hline
\end{tabular}

the desired one. It can be proved that having $h_{13}=0$ and $h_{33}=1$, if $x=0$ or $z=0$ or $\phi=0$ then $\mathbf{H}=\mathbf{I}$. The demonstration is straightforward from (3). Thus, we have in our problem that we finally get the desired pose:

$$
h_{13}=0, h_{33}=1 \text { and } x=0 \Leftrightarrow \mathbf{H}=\mathbf{I} \text {. }
$$

\section{Simulation Results}

Several simulations have been carried out to demonstrate the validity of the approach presented. Performance with image noise and with calibration errors is analyzed. The simulated data is obtained by generating a virtual planar scene consisting of a distribution of random $3 \mathrm{D}$ points. The scene is projected to the image plane using a virtual camera; the size of the virtual images is $640 \times 480$ pixels. In each loop of the control, the homography between the current and target image is computed from the matched points, and the control law sends the velocities $(v, \omega)$ to the robot. The values of the control gains used in the simulations are $k_{13}=1$ and $k_{33}=0.2 ;$ and $T_{1}=40 s, T_{2}=80 s$.

The initial position of the simulations carried out (Fig. 4) is $\left(-5,-15,5^{\circ}\right)$ and the target position is $\left(0,0,0^{\circ}\right)$. Two simulations are shown, one without noise and the other adding white image noise to the points with a standard deviation of $\sigma=0.3$ pixels. The motion of the robot is shown in the $x$ and $z$-coordinates and with the rotation $\phi$; the evolution of $h_{13}$ and $h_{33}$ obtained is also shown. It can be seen that the method converges properly in spite of image noise. In Fig. 4(h) the final position error is shown for different increasing values of the image noise, showing also a good performance with noise.

When the control law matrix is computed, the parameter $\alpha_{x}$ of the calibration matrix is used. Besides, at the beginning of this paper we have assumed that the principal point is in the centre of the image. In Fig. 5 we show the performance of the control to calibration errors. The value of the focal length of the control law has been fixed to $f=6 \mathrm{~mm}$ and then, its real value is modified to see the final position error obtained, Fig. 5(left). In Fig. 5(right) the value of $x_{0}$ used in the control is zero while its real value is changed. Results show that a rough calibration is enough for the convergence.

This approach does not compute the decomposition of the homography, therefore we need to know neither the value of the normal (n) to the plane that generates the homography nor the distance to it $(d)$. As previously explained we

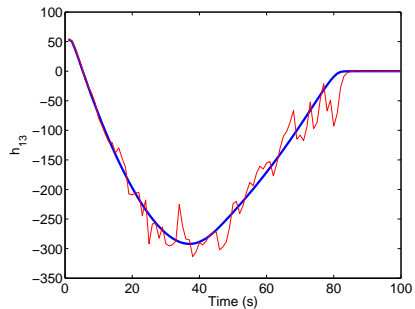

(a) $h_{13}$

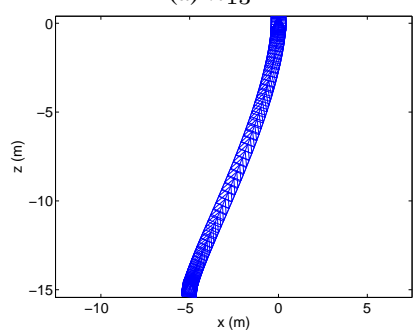

(c) Path without noise

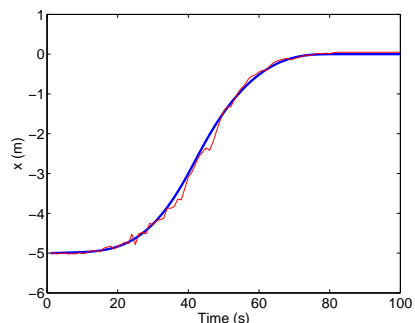

(e) Lateral motion

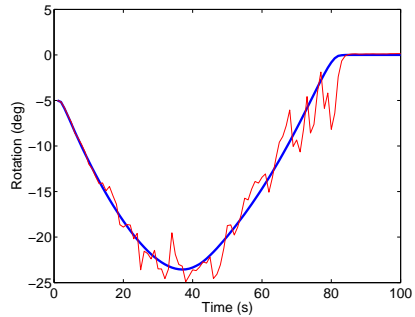

(g) Robot rotation

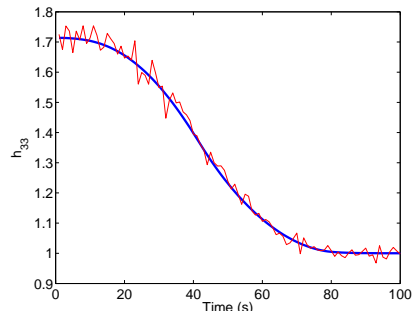

(b) $h_{33}$

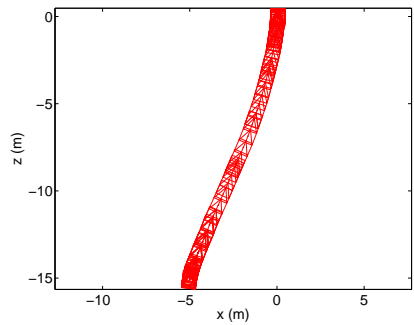

(d) Path with noise

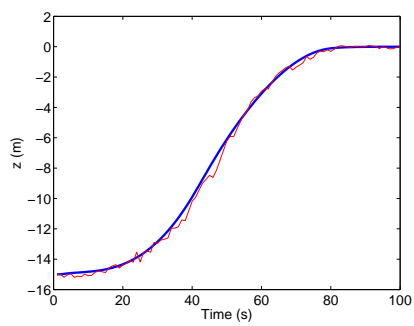

(f) Forward motion

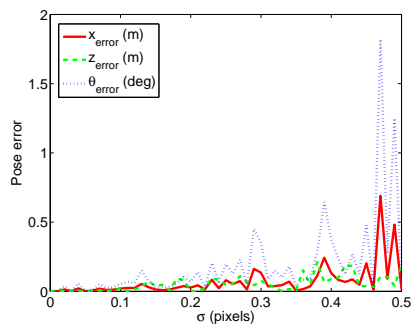

(h) Final error vs image noise
Fig. 4. (a-g) Simulation without noise (thick line) and with image white noise of $\sigma=0.3$ pixels (thin line). (h) Final error varying the image noise.

have considered the parameter $n_{z} / d$ as a constant gain of the control. In Fig. 6 we show the effect of this in the performance of the control. The real distance $d$ from the origin to the plane in these simulations is $15 \mathrm{~m}$., and the real value of $n_{z}$ is -0.69 , while the values used in the control are modified. The results shows that the convergence of the method is not affected and good final position errors are obtained.

\section{CONCLUSIONS}

In this paper we have presented a new homography-based visual control approach that deals with the nonholonomic constraints of the platform. The control law is obtained from the input-output linearization of the system, transforming the visual control problem into a tracking problem where the desired trajectories of the homography elements used as input of the control are defined. This method uses neither 

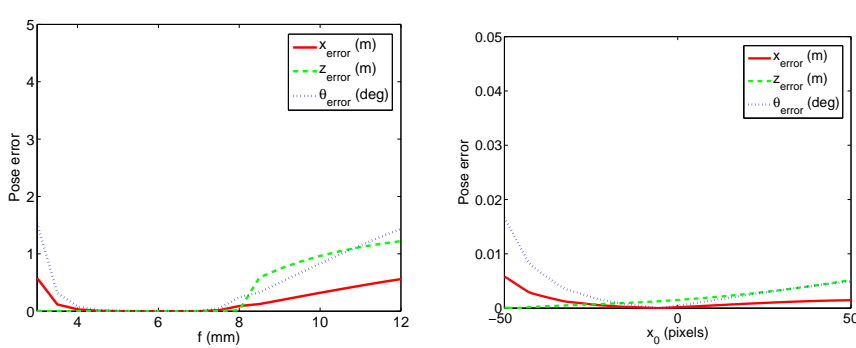

Fig. 5. Final error varying the focal length (left) and varying the position of the principal point (right).
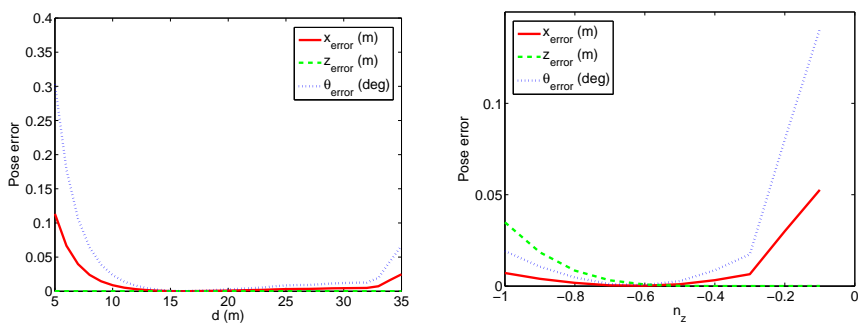

Fig. 6. Final error varying the unknown plane parameters.

the homography decomposition nor any measure of the 3D scene. Simulations show the robustness of the control to image noise and calibration errors.

\section{APPENDIX}

\section{A. Plane Parallax}

In the method presented in this paper we use the plane parallax concept [8]. We describe it to give the reader a self contained explanation. Let us suppose the computed homography $\mathbf{H}$ induced by plane $\pi$ and the projections in both images $\left(\mathbf{p}_{1}, \mathbf{p}_{2}\right)$ of a point $(\mathbf{p})$ not belonging to the plane (Fig. 7). The corresponding point of $\mathbf{p}_{1}$ through the homography $\mathbf{H}$ is $\overline{\mathbf{p}}_{2}=\mathbf{H} \mathbf{p}_{1}$. The line joining $\mathbf{p}_{2}$ to $\overline{\mathbf{p}}_{2}$ is the epipolar line of $\mathbf{p}$ in the second image. Repeating the process with another point $\mathbf{q}$, the line joining $\mathbf{q}_{2}$ to $\overline{\mathbf{q}}_{2}$ is obtained. The intersection of these two lines determine the epipole $\mathbf{e}_{2}$. Once the epipole $\mathbf{e}_{2}$ is computed the fundamental matrix can be obtained using the homography as $\mathbf{F}=\left[\mathbf{e}_{2}\right]_{\times} \mathbf{H}$ where, given the epipole in homogeneous coordinates, $\mathbf{e}_{2}=$ $\left(e_{x}, e_{y}, 1\right)^{T},\left[\mathbf{e}_{2}\right]_{\times}$denotes a $3 \times 3$ skew-symmetric matrix. Then, the epipolar geometry is determined and the epipole in the first image $\mathbf{e}_{1}$ can be computed $\left(\mathbf{F e}_{1}=0\right)$.

Therefore the epipole in the current image can be easily obtained from a homography $\mathbf{H}$ and two points not belonging to the plane of $\mathbf{H}$. In our visual servoing method we call current image the image taken at the current position as the robot moves; the target image is the one taken at the desired position. Then, the epipole in the current and target image are $\mathbf{e}_{c}, \mathbf{e}_{t}$ respectively. As we consider that the robot moves in a planar surface only the $x$-coordinate of the epipoles $\left(e_{c x}\right.$, $\left.e_{t x}\right)$ will be used.

Usually more than two points out of the plane will be available; moreover, we have to take into account the presence of outliers. Therefore a RANSAC algorithm can be used to obtain the best estimation of the epipoles. Once a

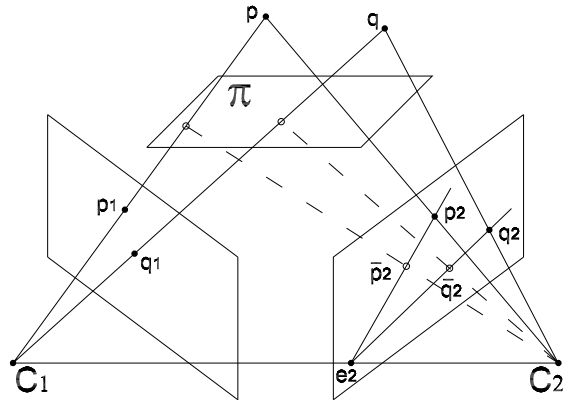

Fig. 7. Parallax relative to a plane.

set of straight lines result as inliers, the epipole is computed as the intersection of these lines. Due to noise they will not intersect exactly in one point, so the Singular Value Decomposition (SVD) is used to get the intersection point.

\section{REFERENCES}

[1] R. Basri, E. Rivlin and I. Shimshoni, "Visual Homing: Surfing on the Epipoles ", International Journal of Computer Vision, vol. 33, no. 2, 1999, 117-137.

[2] S. Benhimane and E. Malis, "Homography-based 2D Visual Servoing", IEEE Int. Conf. on Robotics and Automation, 2006, pp. 2397-2402.

[3] S. Benhimane, E. Malis, P. Rives and J. R. Azinheira, "Vision-based Control for Car Platooning using Homography Decomposition", IEEE Int. Conference on Robotics and Automation, 2005, pp. 2173-2178.

[4] F. Conticelli and B. Allotta, "Nonlinear Controllability and Stability Analysis of Adaptive Image-Based Systems", IEEE Transactions on Robotics and Automation, vol. 17, no. 2, 2001, pp. 208-214.

[5] P. I. Corke and S. A. Hutchinson, "A New Partitioned Approach to Image-Based Visual Servo Control", IEEE Transactions on Robotics and Automation, vol. 17, no. 4, 2001, pp. 507-515.

[6] G. N. DeSouza and A. C. Kak, "Vision for Mobile Robot Navigation: A Survey", IEEE Trans. on Pattern Analysis and Machine Intelligence, vol. 24, no. 2, 2002, pp. 237-267.

[7] Y. Fang, W. E. Dixon, D. M. Dawson and P. Chawda, "Homographybased visual servo regulation of mobile robots", IEEE Transactions on Systems, Man, and Cybernetics, Part B, vol. 35, no. 5, 2005, pp. $1041-1050$

[8] R. I. Hartley and A. Zisserman, "Multiple View Geometry in Computer Vision", Cambridge University Press, 2004.

[9] S. Hutchinson, G. Hager, and P. I. Corke, "A Tutorial on Visual Servo Control", IEEE Transactions on Robotics and Automation, vol. 12, no. 5, Oct. 1996, pp. 651-670.

[10] A. Isidori, Nonlinear Control Systems, Springer, 1995.

[11] G. López-Nicolás and C. Sagüés and J.J. Guerrero and D. Kragic and P. Jensfelt, "Nonholonomic Epipolar Visual Servoing", IEEE Int. Conf. on Robotics and Automation, 2006, pp. 2378-2384.

[12] Y. Ma, J. Kosecka and S. Sastry, "Vision guided navigation for a nonholonomic mobile robot", IEEE Transactions on Robotics and Automation, vol. 15, no. 3, 1999, pp. 521-537.

[13] E. Malis and F. Chaumette, "2 1/2 D Visual Servoing with Respect to Unknown Objects Through a New Estimation Scheme of Camera Displacement", Int. J. Comput. Vision, vol. 37, no. 1, 2000, pp. 79-97.

[14] E. Malis, F. Chaumette and S. Boudet, "2 1/2 D Visual Servoing", IEEE Transactions on Robotics and Automation, vol. 15, no. 2, 1999, pp. 234-246.

[15] P. Rives, "Visual Servoing Based on Epipolar Geometry", IEEE/RSJ International Conference on Intelligent Robots and Systems, vol. 1, 2000, pp. 602-607.

[16] C. Sagüés and J.J. Guerrero, "Visual correction for mobile robot homing", Robotics and Autonomous Systems, vol. 50, no. 1, 2005, pp. 41-49.

[17] J. J. E. Slotine and W. Li, Applied nonlinear control, Prentice Hall, Englewood Cliffs NJ, 1991.

[18] D. P. Tsakiris, P. Rives and C. Samson, "Extending Visual Servoing Techniques to Nonholonomic Mobile Robots", The Confluence of Vision and Control, LNCIS, Springer-Verlag, 1998. 